\title{
Flow-mediated dilation can be used to predict incident hypertension in patients with hyperuricemia
}

Chunli Han'1 Z Zhanchao Xian², Yang Zou², Zhiyong Liao², Rongfeng Yang ${ }^{2}$, Chunxia Zou², Xiaoqing Wang ${ }^{2}$, Yan Sun ${ }^{3}$

${ }^{1}$ Department of Cardiology, the Fifth Subsidiary Sun Yat-sen University Hospital, Zhuhai, China

${ }^{2}$ Department of Cardiology, Shenzhen Sun Yat-sen Cardiovascular Hospital, Shenzhen, China

${ }^{3}$ Department of Endocrinology, Xili People's Hospital of Nanshan District, Shenzhen, China

Submitted: 10 November 2017

Accepted: 30 December 2017

Arch Med Sci 2019; 15, 2: 343-349

DOI: https://doi.org/10.5114/aoms.2018.73856

Copyright $\odot 2018$ Termedia \& Banach

\section{Abstract}

Introduction: The aim of the study was to evaluate whether flow-mediated dilation (FMD) can be used to predict incident hypertension in patients with hyperuricemia.

Material and methods: Normotensive participants with and without hyperuricemia at baseline were prospectively enrolled. Flow-mediated dilation was assessed at baseline, and after 1 year's follow-up the incidence of hypertension was compared between those with and without hyperuricemia. The predictive value of baseline FMD for incident hypertension among hyperuricemia patients was evaluated.

Results: A total of 222 participants were included. Mean systolic and diastolic blood pressure (BP) was $129.5 \pm 8.4 \mathrm{~mm} \mathrm{Hg}$ and $78.3 \pm 7.9 \mathrm{~mm} \mathrm{Hg}$. Mean serum uric acid (UA) level was $4.4 \pm 2.8 \mathrm{mg} / \mathrm{dl}$. Mean FMD was $5.1 \pm 2.7 \%$. Compared to normal UA group, hyperuricemia group had higher proportion of male (58.4\% vs. $61.2 \%)$, higher systolic BP (125.4 $\pm 7.9 \mathrm{~mm} \mathrm{Hg}$ vs. 132.1 $\pm 7.3 \mathrm{~mm} \mathrm{Hg}$ ), serum high sensitivity C-reactive protein $(3.9 \pm 2.2 \mathrm{mg} / \mathrm{dl}$ vs. $4.5 \pm 3.0 \mathrm{mg} / \mathrm{dl})$ and UA $(3.5 \pm 1.4 \mathrm{mg} / \mathrm{dl}$ vs. $5.7 \pm 0.7 \mathrm{mg} / \mathrm{dl})$ levels, but lower mean FMD ( $5.6 \pm 2.4 \%$ vs. $4.8 \pm 2.0 \%)$ ( $p<0.05$ for all comparisons). No participant in normal UA group developed hypertension, while in hyperuricemia group, 6 participants developed hypertension. In hyperuricemia participants, after adjusted for covariates, per 1-standard deviation decrease in baseline FMD remained significantly associated with $15 \%$ increased risk of incident hypertension.

Conclusions: Patients with hyperuricemia have an increased risk of developing hypertension, and low baseline FMD in hyperuricemia patients is associated with significantly increased risk of incident hypertension.

Key words: hypertension, hyperuricemia, flow-mediated dilation.

\section{Introduction}

Hypertension has become the most prevalent chronic disease around the world [1, 2]. In addition, hypertension is a well-known risk factor for target organ damage, cardiovascular diseases (CVD), and all-cause mortality [3-6]. Therefore, effective prevention is essential to reduce the health and economic burden of hypertension $[1,7]$.

\author{
Corresponding authors: \\ Dr. Xiaoqing Wang \\ Department of Cardiology \\ Shenzhen Sun Yat-sen \\ Cardiovascular Hospital \\ 1021 Dongmen Bei Road \\ Guangdong Province \\ 518020 Shenzhen, China \\ E-mail: wangxiaoq1971@163. \\ com \\ Dr. Yan Sun \\ Department \\ of Endocrinology \\ Xili People's Hospital \\ of Nanshan District \\ Guangdong Province \\ 518020 Shenzhen, China \\ E-mail: \\ sun13760431515@163.com
}


Prior observational studies indicate that increased serum uric acid (UA) level is associated with blood pressure (BP) elevation [8-10], and the potential mechanisms are multifactorial and include oxidative stress, systemic inflammation, an endothelial and vascular dysfunction [11-13]. Therefore, one may anticipate that reducing serum UA level to the normal range may be beneficial for hypertension prevention. However, as yet, no solid evidence is available to support this hypothesis, and hypertension guidelines also did not recommend hyperuricemia treatment for primary prevention of hypertension $[1,7]$.

Flow-mediated dilation (FMD) is a sensitive and noninvasive approach for endothelial and vascular function assessment $[14,15]$. Numerous prior longitudinal studies indicate that baseline FMD can be used to predict cardiovascular events in community populations $[16,17]$. However, it is unknown whether FMD is different in patients with hyperuricemia compared to those with a normal serum UA level. In addition, whether baseline FMD can be used to predict incident hypertension in patients with hyperuricemia is also not fully explored yet.

We therefore conducted a prospective study and enrolled normotensive participants with and without hyperuricemia at baseline. After 12 months' follow-up, we compared the incidence of hypertension between these two groups and also evaluated whether baseline FMD can be used to predict incident hypertension in patients with hyperuricemia. From a clinical perspective, such information will not only help elucidate the underlying mechanisms relating hyperuricemia to hypertension development, but will also help in determining whether improving FMD by means of reducing uric acid would be beneficial for preventing hypertension development.

\section{Material and methods}

\section{Study participants}

This study was approved by the Research Ethic Committee of the Fifth subsidiary Sun Yat-sen University Hospital. Informed consent was obtained before enrollment and all studied participants were treated in accordance with the Declaration of Helsinki. The included criteria were: 18-75 years old, clinic systolic (SBP) and diastolic $\mathrm{BP}(\mathrm{DBP})<140$ and $90 \mathrm{~mm} \mathrm{Hg}$ during the screening visit, and currently not on hyperuricemia treatment. The exclusion criteria were: previous antihypertensive treatment or a history of hypertension (either self-reported or documented), hyperuricemia treatment in the past 6 months or a gout flare episode in the past 3 months, histories of coronary heart disease, ischemic stroke, peripheral artery disease, congestive heart failure, or chronic kidney disease (either self-reported or documented).

\section{Baseline data collection}

Baseline data including demographics and medical history were recorded using a structured questionnaire. In brief, current smoking was defined as having smoked a cigarette in the past 30 days. Diabetes mellitus was defined as fasting plasma glucose $(\mathrm{FPG})>126 \mathrm{mg} / \mathrm{dl}$ or current usage of hypoglycemic medications and/or insulin. Clinic BP was measured in accordance with the JNC7 guideline recommendation [7]. Resting BP was measured 3 times after the participant had sat quietly for 5 min (HEM7200, Omron Healthcare, Tokyo, Japan), with the arm supported at heart level and legs placed on the floor. The average of the second and third BP readings was used as clinic BP. Fasting venous blood was used for lipid profile, FPG, creatinine, high-sensitivity C-reactive protein (hs-CRP) and UA level assessments. In brief, estimated glomerular filtration rate (eGFR) was calculated using the Modification of Diet in Renal Disease (MDRD) formula [18].

\section{Brachial FMD assessment}

Brachial FMD was assessed in a fasting state. The participant was supine and the left arm was used to obtain baseline SBP. A standard pediatric cuff was placed around the right arm, nearly 2 inches below the antecubital fossa [15]. A linear-array multi-frequency transducer operating at $10 \mathrm{MHz}$ (GE Logiq 700 Device) was applied to obtain baseline images of the right brachial artery. Two minutes later, the cuff was inflated to $50 \mathrm{~mm} \mathrm{Hg}$ above baseline systolic BP to occlude the right brachial artery for 4 min [15]. Thereafter, the cuff was deflated and images of the right brachial artery were captured continuously for 2 min. The change of diameters was automatically calculated using the formula as follows: (maximum diameter - baseline diameter)/baseline diameter $\times 100 \%$ [15].

\section{Follow-up}

Participants were followed up at the $6^{\text {th }}$ month after the baseline visit via telephone call and at the $12^{\text {th }}$ month at the outpatient clinic. Participants who had an acute gout flare episode or had hyperuricemia treatment during follow-up were excluded from the final analysis, and those with antihypertensive treatment during follow-up were defined as having new-onset hypertension. At the $12^{\text {th }}$ month follow-up, clinic BP was measured in the outpatient clinic, and those with systolic BP 
$>140 \mathrm{~mm} \mathrm{Hg}$ and/or diastolic BP > $90 \mathrm{~mm} \mathrm{Hg}$ within two consecutive days were defined as having new-onset hypertension.

\section{Statistical analysis}

Continuous variables were expressed as mean \pm SD or median (quartiles) as appropriate, and categorical variables were expressed as number and frequency of cases. Between-group differences were evaluated by the independent Student $t$ test or the $\chi^{2}$ analysis as appropriate. Cox proportional hazards regression analysis was used to evaluate the predictive value of baseline FMD for incident hypertension. The hazard ratio (HR) represents the risk associated with a 1 -SD decrease in baseline FMD for incident hypertension. Statistical analysis was conducted in SPSS 23.0 (IBM, USA). All p-values were 2 sided, and statistical significance was defined as $p<0.05$.

\section{Results}

\section{Participants' enrollment}

Between July and September of 2016, a total of 278 participants were screened in the outpatient clinic. Among them, 18 had clinic systolic and/ or diastolic BP > 140/90 mm Hg, 4 had previous antihypertensive treatment, 4 had hyperuricemia treatment in the past 6 months, 3 had a gout flare episode in the past 3 months, 12 had a history of coronary heart disease, ischemic stroke, peripheral artery disease, congestive heart failure, or chronic kidney disease, and 10 did not want to participate in the current study. A total of 227 participants

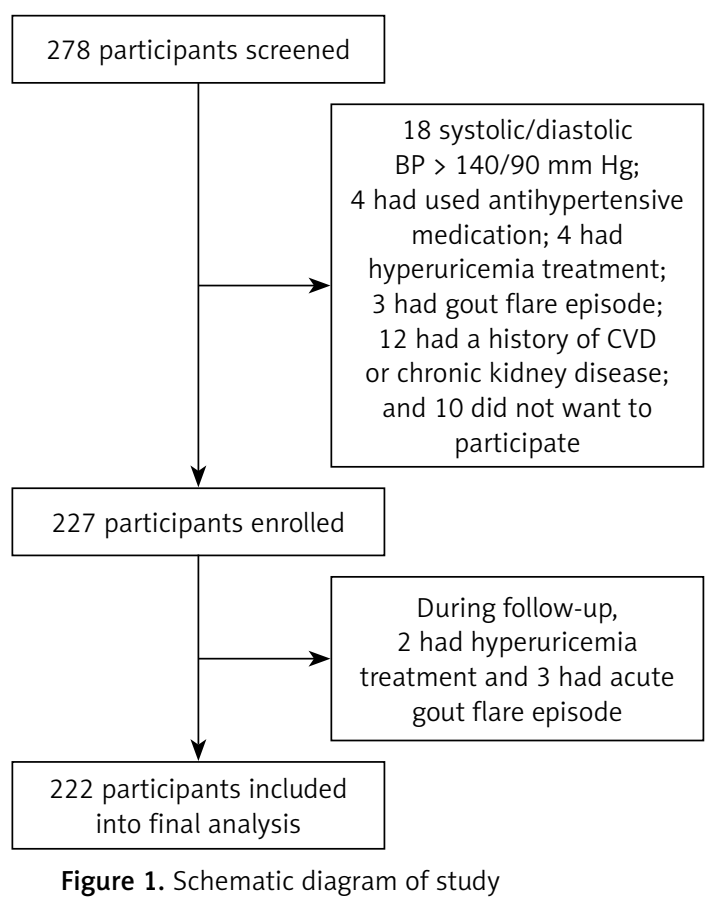

were enrolled. During follow-up, 3 patients had an acute gout flare episode and another 2 had hyperuricemia treatment; therefore, 222 participants were included in the final analysis. A schematic outline of the study is presented in Figure 1.

\section{Baseline characteristics}

The mean age of participants was $46.5 \pm 16.7$ years, male participants accounted for $59.5 \%$, and $39.2 \%$ were current smokers. The mean systolic and diastolic BP were $129.5 \pm 8.4 \mathrm{~mm} \mathrm{Hg}$ and 78.3 $\pm 7.9 \mathrm{~mm} \mathrm{Hg}$, respectively. The mean serum UA level was $4.4 \pm 2.8 \mathrm{mg} / \mathrm{dl}$, and based on the cutoff value $\geq 6 \mathrm{mg} / \mathrm{dl}$ for males and $\geq 5 \mathrm{mg} /$ dl for females [19], $38.3 \%$ of participants were diagnosed with hyperuricemia. The mean FMD was $5.1 \pm 2.7 \%$ (Table I).

Table I. Baseline characteristics $(n=222)$

\begin{tabular}{|c|c|}
\hline Variables & Value \\
\hline Age [years] & $46.5 \pm 16.7$ \\
\hline Male & $132(59.5 \%)$ \\
\hline Smoker & $87(39.2 \%)$ \\
\hline Systolic BP [mm Hg] & $129.5 \pm 8.4$ \\
\hline Diastolic BP [mm Hg] & $78.3 \pm 7.9$ \\
\hline Heart rate $[\mathrm{bpm}]$ & $72.4 \pm 12.5$ \\
\hline Total cholesterol [mmol/l] & $5.0 \pm 1.2$ \\
\hline Triglyceride $[\mathrm{mmol} / \mathrm{l}]^{*}$ & $1.8(0.9-2.3)$ \\
\hline LDL-cholesterol [mmol/l] & $3.2 \pm 1.1$ \\
\hline HDL-cholesterol [mmol/l] & $1.2 \pm 0.5$ \\
\hline $\mathrm{FPG}[\mathrm{mg} / \mathrm{dl}]$ & $123.7 \pm 14.5$ \\
\hline Creatinine $[\mu \mathrm{mol} / \mathrm{l}]$ & $60.5 \pm 10.7$ \\
\hline eGFR $\left[\mathrm{ml} / \mathrm{min} / 1.73 \mathrm{~m}^{2}\right]$ & $95.7 \pm 4.2$ \\
\hline $\mathrm{Hs}-\mathrm{CRP}[\mathrm{mg} / \mathrm{dl}]$ & $4.2 \pm 2.6$ \\
\hline UA [mg/dl] & $4.4 \pm 2.8$ \\
\hline Diabetes mellitus & $65(29.3 \%)$ \\
\hline Dyslipidemia & $72(32.4 \%)$ \\
\hline Hyperuricemia & $85(38.3 \%)$ \\
\hline Aspirin & $70(31.5 \%)$ \\
\hline Statins & $58(26.1 \%)$ \\
\hline Hypoglycemic medication & $58(26.1 \%)$ \\
\hline Insulin & $20(9.0 \%)$ \\
\hline FMD (\%) & $5.1 \pm 2.2$ \\
\hline
\end{tabular}




\section{Comparisons between normal and hyperuricemia groups}

Participants were divided into two groups based on baseline serum UA level. As presented in Table II, compared to those with normal UA level, participants with hyperuricemia were more likely to be male $(58.4 \%$ vs. $61.2 \%)$, had higher baseline systolic BP (125.4 $\pm 7.9 \mathrm{~mm} \mathrm{Hg}$ vs. $132.1 \pm 7.3 \mathrm{~mm} \mathrm{Hg})$, serum hs-CRP $(3.9 \pm 2.2 \mathrm{mg} / \mathrm{dl}$ vs. $4.5 \pm 3.0 \mathrm{mg} / \mathrm{dl})$ and UA $(3.5 \pm 1.4 \mathrm{mg} / \mathrm{dl}$ vs. $5.7 \pm 0.7 \mathrm{mg} / \mathrm{dl})$ levels, and lower mean FMD (5.6 $\pm 2.4 \%$ vs. $4.8 \pm 2.0 \%)$.

\section{Incidence of hypertension}

During 12 months' follow-up, no participant in the normal UA group developed hypertension, while in the hyperuricemia group, 6 (6.9\%) participants developed hypertension. Among them, 1 was diagnosed with hypertension at 10 months' follow-up at the local hospital (SBP/DBP were
146/82 $\mathrm{mm} \mathrm{Hg}$ ) and was treated with an angiotensin converting enzyme inhibitor (lisinopril $20 \mathrm{mg} / \mathrm{qd}$ ), and the other 5 participants were diagnosed with hypertension (mean SBP and DBP were $145.5 \pm 3.2 \mathrm{~mm} \mathrm{Hg}$ and $81.3 \pm 4.9 \mathrm{~mm} \mathrm{Hg}$, respectively) during the outpatient clinic visit at the $12^{\text {th }}$ month follow-up.

\section{Association of baseline FMD and incident hypertension}

In participants with hyperuricemia, in the unadjusted model, 1 - SD decrease in baseline FMD was associated with nearly 2-fold higher risk of incident hypertension (Table III). With stepwise adjustment, after adjustment for potential covariates including age, male gender, smoking, diabetes mellitus, eGFR, baseline systolic BP and hs-CRP, 1 - SD decrease in baseline FMD remained significantly associated with 15\% increased risk of inci-

Table II. Comparisons between normal and hyperuricemia groups

\begin{tabular}{|c|c|c|}
\hline Variables & Normal $(n=137)$ & Hyperuricemia $(n=85)$ \\
\hline Age [years] & $45.3 \pm 15.9$ & $47.9 \pm 17.2$ \\
\hline Male & 80 (58.4\%) & $52(61.2 \%)^{\#}$ \\
\hline Smoker & $54(39.4 \%)$ & $33(38.8 \%)$ \\
\hline Systolic BP [mm Hg] & $125.4 \pm 7.9$ & $132.1 \pm 7.3^{\#}$ \\
\hline Diastolic BP [mm Hg] & $77.6 \pm 6.8$ & $78.9 \pm 7.5$ \\
\hline Heart rate $[\mathrm{bpm}]$ & $73.7 \pm 12.8$ & $72.0 \pm 11.4$ \\
\hline Total cholesterol [mmol/l] & $5.1 \pm 1.4$ & $5.0 \pm 1.1$ \\
\hline Triglyceride $[\mathrm{mmol} / \mathrm{l}]^{*}$ & $1.8(0.8-2.2)$ & $1.8(0.9-2.3)$ \\
\hline LDL-cholesterol [mmol/l] & $3.2 \pm 1.0$ & $3.3 \pm 1.2$ \\
\hline HDL-cholesterol [mmol/l] & $1.1 \pm 0.6$ & $1.2 \pm 0.5$ \\
\hline $\mathrm{FPG}[\mathrm{mg} / \mathrm{dl}]$ & $121.4 \pm 14.2$ & $124.6 \pm 15.0$ \\
\hline Creatinine $[\mu \mathrm{mol} / \mathrm{l}]$ & $58.7 \pm 10.3$ & $61.4 \pm 11.7$ \\
\hline $\mathrm{eGFR}\left[\mathrm{ml} / \mathrm{min} / 1.73 \mathrm{~m}^{2}\right]$ & $96.1 \pm 5.2$ & $94.5 \pm 4.3$ \\
\hline $\mathrm{Hs}-\mathrm{CRP}[\mathrm{mg} / \mathrm{dl}]$ & $3.9 \pm 2.2$ & $4.5 \pm 3.0^{\#}$ \\
\hline $\mathrm{UA}[\mathrm{mg} / \mathrm{dl}]$ & $3.5 \pm 1.4$ & $5.7 \pm 0.7^{\#}$ \\
\hline Diabetes mellitus & $40(29.2 \%)$ & $25(29.4 \%)$ \\
\hline Dyslipidemia & $44(32.1 \%)$ & $28(32.9 \%)$ \\
\hline Aspirin & $42(30.7 \%)$ & $28(32.9 \%)$ \\
\hline Statins & $36(26.3 \%)$ & $22(25.9 \%)$ \\
\hline Hypoglycemic medication & $35(25.5 \%)$ & $23(27.1 \%)$ \\
\hline Insulin & $12(8.8 \%)$ & $8(9.4 \%)$ \\
\hline FMD (\%) & $5.6 \pm 2.4$ & $4.8 \pm 2.0^{\#}$ \\
\hline
\end{tabular}


dent hypertension, indicating that in hyperuricemia patients, baseline FMD can be used to predict the 1-year incidence of hypertension.

\section{Interaction between risk factors and baseline FMD for incident hypertension}

Participants with hyperuricemia were divided into 3 groups based on tertile baseline FMD values and the upper tertile was defined as the reference group. As presented in Table IV, in those $\geq 60$ years, male participants, with baseline systolic BP $\geq 130 \mathrm{~mm} \mathrm{Hg}$ and hs-CRP level $\geq 3 \mathrm{mg} / \mathrm{dl}$, reduced baseline FMD was associated with 16\%, 8\%, 12\%, and $9 \%$ increased incidence of hypertension (lower tertile versus upper tertile). The interaction between potential risk factors and baseline FMD is significant, indicating that ageing, male gender, higher baseline systolic BP, or higher hs-CRP level might potentiate the effect of reduced FMD on hypertension development.

\section{Discussion}

To our best knowledge, the current study is one of the first few studies to investigate the association of baseline FMD and incident hypertension in patients with hyperuricemia. The principal findings include: first, compared to those with normal serum UA level, patients with hyperuricemia had significantly reduced FMD; second, baseline FMD can be used to independently predict hypertension development in hyperuricemia patients; third, decreased FMD was associated with incident hypertension, especially in those with older age, males, with higher baseline systolic BP and
Table III. Association of baseline FMD and incident hypertension

\begin{tabular}{|lccc|}
\hline Variable & Hazard ratio & $95 \% \mathrm{Cl}$ & $P$-value \\
\hline Unadjusted & 2.04 & $1.85-2.33$ & $<0.001$ \\
\hline Model 1 & 1.86 & $1.62-2.02$ & $<0.001$ \\
\hline Model 2 & 1.41 & $1.25-1.83$ & 0.007 \\
\hline Model 3 & 1.15 & $1.07-1.32$ & 0.016 \\
\hline
\end{tabular}

Model 1 -adjusted for age and male gender, Model 2 - further adjusted for smoking, diabetes mellitus, estimated glomerular filtration rate and hs-CRP, Model 3 - further adjusted for baseline systolic $\mathrm{BP}, \mathrm{Cl}$ - confidence interval.

increased serum hs-CRP level. The clinical implication of our current study is that in patients with hyperuricemia, measuring FMD at baseline could be used to predict the incidence of hypertension. In addition, it can also help raise both physicians' and patients' awareness of their potential risk of developing hypertension, which in turn may promote primary prevention.

In recent decades, numerous observational studies have indicated that increased serum UA level was associated with hypertension, diabetes mellitus and CVD events. For example, Sundström et al. [20] reported that in 3329 Framingham Study participants, a 1 - SD higher serum UA was associated with $17 \%$ higher risk of developing hypertension. In another study, Shankar et al. [21] also reported that increasing quartiles of serum UA level was independently associated with 10-year incidence of hypertension among community-dwelling older adults. Dehghan et al. [22] enrolled 4536 subjects free from diabetes at baseline. During

Table IV. Interaction between potential risk factors and baseline FMD for incident hypertension

\begin{tabular}{|c|c|c|c|c|}
\hline \multirow[t]{2}{*}{ Parameter } & \multicolumn{3}{|c|}{ FMD } & \multirow{2}{*}{$\begin{array}{c}P \text {-value } \\
\text { (interaction) }\end{array}$} \\
\hline & T3 (5.5-6.8\%) & T2 (4.2-5.4\%) & T1 (2.8-4.1\%) & \\
\hline$<60$ years & Ref & $1.06(0.94-1.11)$ & $1.11(0.98-1.22)$ & $<0.001$ \\
\hline$\geq 60$ years & Ref & $1.09(0.97-1.15)$ & $1.16(1.02-1.24)$ & \\
\hline Female & Ref & $0.98(0.92-1.14)$ & $1.01(0.95-1.09)$ & 0.031 \\
\hline Male & Ref & $1.04(0.95-1.13)$ & $1.08(1.01-1.19)$ & \\
\hline Non-smoker & Ref & $0.95(0.89-1.04)$ & $0.97(0.93-1.07)$ & 0.165 \\
\hline Smoker & Ref & $1.02(0.97-1.12)$ & $1.06(0.99-1.14)$ & \\
\hline Systolic BP < $130 \mathrm{~mm} \mathrm{Hg}$ & Ref & $1.04(0.97-1.16)$ & $1.07(0.99-1.19)$ & $<0.001$ \\
\hline Systolic BP $\geq 130 \mathrm{~mm} \mathrm{Hg}$ & Ref & $1.06(0.99-1.18)$ & $1.12(1.06-1.23)$ & \\
\hline $\mathrm{eGFR}<90 \mathrm{ml} / \mathrm{min} / 1.73 \mathrm{~m}^{2}$ & Ref & $1.01(0.90-1.06)$ & $1.03(0.95-1.10)$ & 0.306 \\
\hline $\mathrm{eGFR} \geq 90 \mathrm{ml} / \mathrm{min} / 1.73 \mathrm{~m}^{2}$ & Ref & $0.98(0.92-1.11)$ & $1.01(0.95-1.14)$ & \\
\hline $\mathrm{Hs}-\mathrm{CRP}<3 \mathrm{mg} / \mathrm{dl}$ & Ref & $0.96(0.90-1.02)$ & $0.99(0.93-1.08)$ & 0.028 \\
\hline $\mathrm{Hs}-\mathrm{CRP} \geq 3 \mathrm{mg} / \mathrm{dl}$ & Ref & $1.02(0.98-1.15)$ & $1.09(1.02-1.17)$ & \\
\hline
\end{tabular}


a mean of 10.1 years' follow-up, participants in the upper quartile of baseline serum UA level were $68 \%$ more likely to develop type 2 diabetes than were those in the lowest quartile. With respect to the association of UA and cardiovascular events, Alderman et al. [23] reported that among 7978 mild-to-moderate hypertensive participants, despite BP control, serum UA level increase was significantly and directly associated with CVD events. Using the data from the NHANES I epidemiologic follow-up study, Fang et al. [24] reported that increased serum UA levels were independently and significantly associated with risk of cardiovascular mortality. These findings combined indicate that serum UA level could be used to predict hypertension and CVD events.

Consistent with previous reports, our current study also indicated that compared to those with a normal serum UA level, participants with hyperuricemia had higher incidence of hypertension during 1 year's follow-up. The novel finding of our current study is that we found that in hyperuricemia patients, reduced baseline FMD was independently associated with incident hypertension, indicating that baseline FMD may be used to predict BP progress and hypertension development among patients with hyperuricemia. One potential mechanism is that an increased serum UA level elicits oxidative stress and inflammation, which in turn causes nitric oxide reduction and vascular dysfunction [11, 12, 25]. Impaired vasodilation leads to peripheral artery resistance increase and BP elevation [26]. Future randomized controlled trials are needed to evaluate whether improvement of FMD through lifestyle modification or medications could help to prevent hypertension in patients with hyperuricemia.

Interestingly and importantly, we observed that the association of baseline FMD and hypertension development was particularly important in elderly and male participants and those with increased baseline systolic BP and serum hs-CRP levels. Notably, ageing and increased baseline systolic BP are two established risk factors for hypertension development [27]. Male gender is also an independent predictor of hypertension [28]. Increased serum hs-CRP level is associated with endothelial and vascular dysfunction, which in turn could lead to vasoconstriction and BP elevation [29]. The clinical implication of the current findings is that among hyperuricemia patients, those with reduced FMD and additional comorbidities such as ageing, the risk of developing hypertension is much higher than in their younger counterparts, and intensive modification of these risk factors may help to prevent hypertension.

The strengths of the current study are the prospective design and having no loss to follow-up. There were some limitations of the current study: first of all, hypertension diagnosis was based solely on clinic BP. Although clinic BP remains the main approach to diagnosing hypertension, currently, some guidelines recommend 24-hour ambulatory blood pressure monitoring to confirm newly diagnosed hypertension [30]. Secondly, the duration of follow-up was relatively short and we did not evaluate the changes of serum UA level and FMD over time. Thirdly, notably, the hs-CRP level was higher in the hyperuricemia group. Although we adjusted for hs-CRP in the regression model, residual bias might still exist. Last but not least, body mass index (BMI) is an important marker linking hyperuricemia and hypertension. However, we did not collect the BMI data and residual bias might exist and influence the association of hyperuricemia and hypertension development. A future prospective study is warranted to collect the BMI data, which could help reduce residual bias.

In conclusion, our current study indicates that patients with hyperuricemia have a higher risk of developing hypertension compared to those with a normal serum UA level. Low baseline FMD in patients with hyperuricemia is associated with significantly increased risk of incident hypertension.

\section{Acknowledgments}

We thank Fang Yang, PhD, for assisting us in performing statistical analysis of our study.

The study was supported by grants from the Science and Technology project of Shenzhen city of China (JCYJ20170413114916687).

\section{Conflict of interest}

The authors declare no conflict of interest.

\section{References}

1. Mancia G, Fagard R, Narkiewicz K, et al. 2013 ESH/ESC Guidelines for the management of arterial hypertension: the Task Force for the management of arterial hypertension of the European Society of Hypertension (ESH) and of the European Society of Cardiology (ESC). J Hypertens 2013; 31: 1281-357.

2. James PA, Oparil S, Carter BL, et al. 2014 evidence-based guideline for the management of high blood pressure in adults: report from the panel members appointed to the Eighth Joint National Committee (JNC 8). JAMA 2014; 311: 507-20.

3. Thomopoulos C, Parati G, Zanchetti A. Effects of blood pressure lowering on outcome incidence in hypertension: 4. Effects of various classes of antihypertensive drugs: overview and meta-analyses. J Hypertens 2015; 33: 195-211.

4. Xie X, Atkins E, Lv J, et al. Effects of intensive blood pressure lowering on cardiovascular and renal outcomes: updated systematic review and meta-analysis. Lancet 2016; 387: 435-43.

5. Drawz PE, Pajewski NM, Bates JT, et al. Effect of intensive versus standard clinic-based hypertension management on ambulatory blood pressure: results from 
the SPRINT (Systolic Blood Pressure Intervention Trial) Ambulatory Blood Pressure Study. Hypertension 2017; 69: 42-50.

6. Cai A, Zhong Q, Liu C, et al. Associations of systolic and diastolic blood pressure night-to-day ratios with atherosclerotic cardiovascular diseases. Hypertens Res 2016; 39: 874-8.

7. Chobanian AV, Bakris GL, Black HR, et al. The Seventh Report of the Joint National Committee on Prevention, Detection, Evaluation, and Treatment of High Blood Pressure: the JNC 7 report. JAMA 2003; 289: 2560-72.

8. Borghi C, Rosei EA, Bardin T, et al. Serum uric acid and the risk of cardiovascular and renal disease. J Hypertens 2015; 33: 1729-41; discussion 1741.

9. Viazzi F, Parodi D, Leoncini G, et al. Serum uric acid and target organ damage in primary hypertension. Hypertension 2005; 45: 991-6.

10. Ghomari-Boukhatem H, Bouchouicha A, Mekki K, Chenni K, Belhadj M, Bouchenak M. Blood pressure, dyslipidemia and inflammatory factors are related to body mass index in scholar adolescents. Arch Med Sci 2017; 13: 46-52.

11. Kanellis J, Watanabe S, Li JH, et al. Uric acid stimulates monocyte chemoattractant protein-1 production in vascular smooth muscle cells via mitogen-activated protein kinase and cyclooxygenase-2. Hypertension 2003; 41 : 1287-93.

12. Bavishi C, Messerli FH, Rimoldi SF. Serum uric acid in primary hypertension: from innocent bystander to primum movens. Hypertension 2016; 67: 845-7.

13. Faridi KF, Lupton JR, Martin SS, et al. Vitamin D deficiency and non-lipid biomarkers of cardiovascular risk. Arch Med Sci 2017; 13: 732-7.

14. Najjar SS, Scuteri A, Shetty V, et al. Pulse wave velocity is an independent predictor of the longitudinal increase in systolic blood pressure and of incident hypertension in the Baltimore Longitudinal Study of Aging. J Am Coll Cardiol 2008; 51: 1377-83.

15. Harris RA, Nishiyama SK, Wray DW, Richardson RS. UItrasound assessment of flow-mediated dilation. Hypertension 2010; 55: 1075-85.

16. Yeboah J, Crouse JR, Hsu FC, Burke GL, Herrington DM. Brachial flow-mediated dilation predicts incident cardiovascular events in older adults: the Cardiovascular Health Study. Circulation 2007; 115: 2390-7.

17. Yeboah J, Folsom AR, Burke GL, et al. Predictive value of brachial flow-mediated dilation for incident cardiovascular events in a population-based study: the multi-ethnic study of atherosclerosis. Circulation 2009; 120: 502-9.

18. K/DOQI clinical practice guidelines for chronic kidney disease: evaluation, classification, and stratification. Am J Kidney Dis 2002; 39 (2 Suppl 1): S1-266.

19. Bardin T, Richette P. Definition of hyperuricemia and gouty conditions. Curr Opin Rheumatol 2014; 26: 186-91.

20. Sundström J, Sullivan L, D'Agostino RB, Levy D, Kannel WB, Vasan RS. Relations of serum uric acid to longitudinal blood pressure tracking and hypertension incidence. Hypertension 2005; 45: 28-33.

21. Shankar A, Klein R, Klein BE, Nieto FJ. The association between serum uric acid level and long-term incidence of hypertension: population-based cohort study. J Hum Hypertens 2006; 20: 937-45.

22. Dehghan A, van Hoek M, Sijbrands EJ, Hofman A, Witteman JC. High serum uric acid as a novel risk factor for type 2 diabetes. Diabetes Care 2008; 31: 361-2.

23. Alderman MH, Cohen H, Madhavan S, Kivlighn S. Serum uric acid and cardiovascular events in successfully treated hypertensive patients. Hypertension 1999; 34: 144-50.

24. Fang J, Alderman MH. Serum uric acid and cardiovascular mortality the NHANES I epidemiologic follow-up study, 1971-1992. National Health and Nutrition Examination Survey. JAMA 2000; 283: 2404-10.

25. Kanabrocki EL, Third JL, Ryan MD, et al. Circadian relationship of serum uric acid and nitric oxide. JAMA 2000; 283: 2240-1.

26. Messerli FH, Frohlich ED, Dreslinski GR, Suarez DH, Aristimuno GG. Serum uric acid in essential hypertension: an indicator of renal vascular involvement. Ann Intern Med 1980; 93: 817-21.

27. Fields LE, Burt VL, Cutler JA, Hughes J, Roccella EJ, Sorlie $\mathrm{P}$. The burden of adult hypertension in the United States 1999 to 2000: a rising tide. Hypertension 2004; 44: 398-404.

28. Egan BM, Li J, Hutchison FN, Ferdinand KC. Hypertension in the United States, 1999 to 2012: progress toward Healthy People 2020 goals. Circulation 2014; 130: 1692-9.

29. Abdel-Zaher AO, Elkoussi AE, Abudahab LH, Elbakry MH, Elsayed EA. Effect of simvastatin on the antihypertensive activity of losartan in hypertensive hypercholesterolemic animals and patients: role of nitric oxide, oxidative stress, and high-sensitivity C-reactive protein. Fundam Clin Pharmacol 2014; 28: 237-48.

30. Ritchie LD, Campbell NC, Murchie P. New NICE guidelines for hypertension. BMJ 2011; 343: d5644. 